$\check{S}$

M|R|S Internet Journal Nitride Semiconductor Research

\title{
Free-carrier effects and optical phonons in GaNAs/GaAs superlattice heterostructures measured by infrared spectroscopic ellipsometry
}

\author{
J. Šik ${ }^{14}$, M. Schubert ${ }^{12}$, T. Hofmann ${ }^{2}$ and V. Gottschalch ${ }^{3}$ \\ ${ }^{1}$ Center for Microelectronic and Optical Materials Research and Department of Electrical Engineering, \\ University of Nebraska-Lincoln, \\ ${ }^{2}$ Fakultaet fuer Physik und Geowissenschaften, Arbeitsgruppe Halbleiterphysik,University Leipzig, \\ ${ }^{3}$ Fakultaet fuer Chemie und Mineralogie, University Leipzig, \\ ${ }^{4}$ Department of Solid State Physics and Laboratory of Thin FIlms and Nanostructures, Faculty of Science, \\ Masaryk University Brno,
}

(Received Thursday, March 2, 2000; accepted Friday, April 21, 2000)

\begin{abstract}
The infrared-optical properties of GaAs/ $\mathrm{GaN}_{x} \mathrm{As}_{1-x}$ superlattice (SL) heterostructures $(0<x<3.3 \%)$ are studied by variable angle-of-incidence infrared spectroscopic ellipsometry (IRSE) for wavenumbers from $250 \mathrm{~cm}^{-1}$ to $700 \mathrm{~cm}^{-1}$. The undoped SL structures where grown on top of a 300 $\mathrm{nm}$ thick undoped GaAs buffer layer on Te-doped (001) GaAs substrates by metal-organic vapor phase epitaxy (MOVPE). We observe the well-known Berreman-polariton effect within the GaAs LO-phonon region. We further observe a strong polariton-like resonance near the coupled longitudinal-optical plasmon-phonon frequency of the Te-doped substrate at $306 \mathrm{~cm}^{-1}$. For analysis of the IRSE data we employ the harmonic oscillator dielectric function model and the Drude model for free-carrier response. The additional resonance feature is explained by pseudo surface polariton (PSP) interface modes between the Te-doped GaAs and the undoped GaAs buffer layer / SL film. We find that the PSP modes are extremely sensitive to free-carrier properties within the SL structures, and we obtain a strong increase in free-carrier concentration within the GaNAs SL sublayers with increasing $x$ from analysis of the IRSE data. We further observe the localized vibrational modes of nitrogen at $470 \mathrm{~cm}^{-1}$ in the $\mathrm{GaN}_{x} \mathrm{As}_{1-x}$ SL sublayers with a polar strength that increases linearly with $x$, and which can be used to monitor the nitrogen concentration in $\mathrm{GaN}_{x} \mathrm{As}_{1-x}$.
\end{abstract}

\section{Introduction}

The nitrogen-doped low band-gap A(III)-B(V) alloys of $\mathrm{GaN}_{x} \mathrm{As}_{1-x}$ have recently attracted much attention due to wide-range band-gap properties. The large field of possible optoelectronic applications includes optical interconnections, fast switching systems, and low-band-gap detectors [1]. The large chemical and size differences between $\mathrm{N}$ and As causes strongly nonlinear dependence of the band-gap on the composition in this alloy system, and anomalously large optical bowing coefficients have been predicted [2], [3]. Due to the strong lattice mismatch between $\mathrm{GaAs}$ and $\mathrm{GaN}_{x} \mathrm{As}_{1-x}$ for increasing nitrogen incorporation the critical thickness for pseudomorphic $\mathrm{GaN}_{x} \mathrm{As}_{1-x}$ growth decreases rapidly.
SL structures to compensate or stabilize mismatchinduced strain in $\mathrm{GaN}_{x} \mathrm{As}_{1-x}$ epilayers may present a way out of the critical-thickness limitation for single epilayers.

Studies of the optical properties of $\mathrm{GaN}_{x} \mathrm{As}_{1-x}$ have mostly concentrated on photoluminescence and nearinfrared (NIR) transmission investigations of the bandgap dependence versus the nitrogen concentration in single epitaxial layers [4], [5], [6], [7], [8], [9], and superlattice (SL) structures [10]. We found only two Raman investigation reports concerning the phonon properties of $\mathrm{GaN}_{x} \mathrm{As}_{1-x}$ [11], [12], and we are not aware of any existing infrared spectroscopy result. 
Infrared spectroscopic ellipsometry (IRSE) is a powerful technique for nondestructive optical characterization of surfaces, interfaces and thin films [13]. IRSE was demonstrated as a highly sensitive technique to study the Berreman-polariton effect [14] in insulating thin films on metals [15], and in semiconductor heterostructures [16]. The Berreman-polariton effect can be used to precisely measure the spectral locations of the longitudinal-optical (LO) frequencies of polar dielectric thin-film materials. (This effect is actually an "optical wave guide" effect occurring near to the LO frequency, and not the resonant absorption of incident field amplitudes by LO phonons.) We have recently applied IRSE as novel tool to study phonon properties, free-carrier parameters, and strain in group-III-nitride heterostructures grown on sapphire [17], [18], [19].

In this report we present and discuss room temperature IRSE investigations of GaAs/ $\mathrm{GaN}_{x} \mathrm{As}_{1-x}$ SL structures grown by MOVPE. All samples were studied previously by spectroscopic ellipsometry for photon energies from $0.75 \mathrm{eV}$ to $1.55 \mathrm{eV}$ (NIR) [20]. We extracted the complex index of refraction and the fundamental direct band-to-band transition energies $E_{0}$ of the $\mathrm{GaN}_{x} \mathrm{As}_{1-x}$ SL sublayers from the ellipsometry data employing standard critical-point dielectric function models. We observed the characteristic red shift of the $E_{0}$ position with increasing $x$, and the decrease of the $E_{0}$ transition amplitude. From the present IR study of our samples we obtain that the $\mathrm{GaN}_{x} \mathrm{As}_{1-x}$ SL sublayers contain free carriers, which may originate from misfitdislocation-induced or nitrogen-induced donor or acceptor states within the SL structure. However, from the IR response we are not able to differentiate the type of conductivity $(n$ or $p$ ). We report the optically determined free-carrier concentrations within the substrate and the $\mathrm{GaN}_{x} \mathrm{As}_{1-x}$ sublayers, and the frequency, broadening, and amplitude of the nitride-related lattice mode as a function of the nitrogen concentration $x$ for $0 \leq x \leq 3.3$ $\%$. We further discuss the physical origin of the polariton effects in our data within the standard polariton concept.

\section{Theory}

\subsection{Ellipsometry}

The IRSE parameters $\Psi$ and $\Delta$ are defined by the complex ratio of the $p$ - and $s$-polarized reflectance coefficients $r_{p}$ and $r_{s}$, respectively [13]

$$
\rho \equiv \frac{r_{p}}{r_{s}}=\tan \Psi \exp i \Delta
$$

and depend on the angle of incidence $\Phi_{a}$, the thickness $d$ of each layer, and the dielectric functions $\varepsilon_{j}$ of all materials from the heterostructure. Ellipsometry is an indirect technique and model calculations are needed to extract information from individual constituents. Nonlinear regression algorithms are used to vary physically significant model parameters until measured and calculated spectra match as closely as possible. Parametric dielectric function models can greatly reduce the number of free parameters during data analysis. Details and issues of IR ellipsometry data analysis have been extensively discussed elsewhere, and will not be repeated here. (See references [13], [17], [21], [22], and references therein.) The model approach for the infrared response of polar semiconductors with free carriers used in the present work is the same as that discussed in Ref. [18]. The so-called four-parameter semi-quantum model was used in Ref. [18] where $\omega_{\mathrm{TO} i}, \omega_{\mathrm{LO} i}, \gamma_{\mathrm{TO} i}$, and $\gamma_{\mathrm{LO} i}$ are the transverse optical (TO), and longitudinal optical LO-phonon frequencies and broadening parameters, respectively. The free-carrier contribution can be parameterized through the carrier concentration $n$, the carrier effective mass $m^{*}$, and the carrier mobility $\mu$ (See Equations 2 and 3 in Ref. [18].) The high-frequency dielectric constant is $\varepsilon_{\infty}$. Eqs.(2)-(4) in Ref. [18] referred to a uniaxial material. However, in the present work the optical response parallel and perpendicular to the sample surface is treated as isotropic despite the tetragonal distortion of the $\mathrm{GaN}_{x} \mathrm{As}_{1-x}$ SL sublayers due to the tensile strain within the pseudomorphically grown heterostructures. Because we have not observed deviation from the cubic lattice response, we will not consider the uniaxial perturbation of the $\mathrm{GaN}_{x} \mathrm{As}_{1-x}$ SL sublayers parallel to the strain direction. We also did not observe anharmonicity of the lattice resonances, and we have set $\Gamma=\gamma_{\mathrm{TO}}$ $=\gamma_{\mathrm{LO}}$ throughout $[23]$.

\subsection{Surface Polaritons}

Surface polaritons (SP) are excitation states of transverse magnetic (TM) character at the boundary of two media whose dielectric functions fulfill certain conditions [13], [24]. The polariton wave vector is greater than the corresponding vector in the ambient medium. Normally, SP excitation by a simple reflection experiment is not possible, and gratings have to be ruled onto the surface, or light needs to be coupled in upon total reflection at the base of a high index prism [24]. However, it was pointed out by Röseler that under certain circumstances excitation of SP modes can be observed by IRSE at the interface between a polar and a metallic material [13]. There, the fact that the refractive index $N$ $=\sqrt{ } \varepsilon$ in the polar medium becomes less than unity near the LO phonon frequency was discussed as the condi- 
tion at which excitation of the SP mode at the metal interface can happen. The polar film primarily plays the role of the low-index gap for a prism setup, and the "prism" index of refraction is that of the ambient air.

The dispersion relation for SP modes at the boundary between two media, i.e., the condition for TM modes, follows directly from Maxwell, and can be written as follows [24]

$$
\frac{\epsilon_{1}}{\kappa_{1}}=-\frac{\epsilon_{2}}{\kappa_{2}},
$$

with $\kappa_{1,2}=\sqrt{ }\left(k_{x}{ }^{2}-\varepsilon_{1,2}\right)$ being real and positive for a TM wave which is evanescent on both sides of the interface. $\varepsilon_{1}$ and $\varepsilon_{2}$ are the dielectric functions of film and substrate, respectively, and $k_{x}=N_{\mathrm{a}} \sin \left(\Phi_{a}\right)$ is the $x$-component of the incident wave vector (angle of incidence $\Phi_{a}$; ambient index of refraction $N_{a}$ ). The condition for the existence of a "true" SP follows immediately: One of the media must have a negative dielectric function in order to satisfy the equation above [13] [24]. This condition is fulfilled for the Berreman polariton (BP) observed in thin dielectric films attached to a metal surface, and was described in Berreman's original paper [14].

A different type of solution for TM waves with wavevector along the interface can be read from the equation above when both $\varepsilon_{1}$ and $\varepsilon_{2}$ are positive, but one $\kappa$ is imaginary and positive, and the other $\kappa$ is imaginary and negative. We will call this type of TM wave a pseudo surface polariton (PSP) because the wave is not evanescent on both sides of the interface, but presents a power flow transport along the interface similar to that of a "true" SP. This situation is actually often observed (although not referred to in the manner just described) because the Berreman-effect in dielectric films attached to dielectric materials (e.g., a free-standing semiconductor material film) belongs to this second type of TM wave solution. (For the free standing film near its LO frequency neither side of the film interfaces possesses negative dielectric function values. More details of this discussion will be given somewhere else [25].)

The samples investigated here consist in principle of two identical polar materials except for the concentration of free carriers. For now we will treat the films deposited on the doped substrate as one single GaAs film, which combines all layers including the $\mathrm{GaN}_{x} \mathrm{As}_{1-x}$ layers. Note that the $\mathrm{Ga}-\mathrm{N}$ related contributions to the $\mathrm{GaN}_{x} \mathrm{As}_{1-x}$ IR dielectric functions are very small, and can be omitted in the meantime. The dielectric functions $\varepsilon_{1}$ (the undoped GaAs film) and $\varepsilon_{2}$ (the doped GaAs substrate) differ then by the Drude term contribution to $\varepsilon_{2}$ only. These subtle differences lead to well-defined branches of PSP modes, which are related to the occurrence of the coupled plasmon-phonon bulk modes within the substrate $\left(\varepsilon_{2}\right)$. We therefore refer to the PSP modes as surface-plasmon-phonon induced pseudo polaritons. Figure 1 presents the solution of Equation (2) for positive and imaginary $\kappa_{1}$ but negative and imaginary $\kappa_{2}$ as a function of the substrate free carrier concentration $n$ for $\Phi_{a}=70^{\circ}$. For simplicity, and only for now, we assume no broadening $\left(\Gamma_{1}=\Gamma_{2}=0, \mu_{2}=+\infty\right)$.

We find two branches $\left(\mathrm{PSP}^{+}, \mathrm{PSP}^{-}\right)$of PSP modes at spectral positions where the substrate material has an index of refraction of less than 1. The PSP modes follow closely those of the longitudinal-optical coupled plasmon-phonon modes (LPP) in polar semiconductors with free carriers [26], [23]. However, the PSP frequencies are slightly larger than the LPP modes, and depend on the angle of incidence. The inset in Figure 1 shows the wavenumber differences between LPP and PSP modes for both branches as a function of $n$.

The BP is also present in our sample situation because the $\mathrm{BP}$ is one solution of Equation (2). $\varepsilon_{2}$ is negative near the GaAs-LO frequency in the substrate because of the free-carrier coupling, and the combined GaAs film has a positive $\varepsilon_{1} . \kappa_{1}$ and $\kappa_{2}$ are real and positive and the BP is a "true" polariton in the case observed here. The occurrence of the BP was discussed and interpreted in several other publications (See, e.g., Refs. [13], [15], [27], [25]), and will not be further addressed here.

\section{Experimental}

Three samples were grown by MOVPE on Te-doped (001) GaAs using trimethylgallium (TMGa), tertiarybutylarsine (TBAs), and dimethylhydrazine (DMHy). The growth temperature for all samples was $525^{\circ} \mathrm{C}$. The nitrogen incorporation was controlled by the partial TBAs and DMHy pressure values. The partial pressure of TMGa was kept constant. The nitrogen content and the lattice constants were determined by high-resolution XRD using the (004), (115) and (-1-15) reflex pattern. The average parallel misfit for the MQW-SL structures is less than $-1.5 \times 10^{-4}$. The GaAs/GaN $\mathrm{As}_{1-x} \mathrm{SL}$ structures consist of twenty periods of nominally $\sim 9 \mathrm{~nm}$ thick GaAs and $\sim 8 \mathrm{~nm}$ thick $\mathrm{GaN}_{x} \mathrm{As}_{1-x}$ sublayers. The SL structures were grown on top of a $\sim 300 \mathrm{~nm}$ thick GaAs buffer layer, and covered by a $30 \mathrm{~nm}$ thick cap layer. Transmission electron microscopy (TEM), and x-ray diffraction (XRD) investigations were performed to ensure structural quality, and low in-plane lattice mismatch within our samples studied here. NIR SE analysis 
revealed the well-known red shift of the fundamental band-to-band transition $E_{0}$ versus $x$ within the $\mathrm{GaN}_{x} \mathrm{As}_{1}$ ${ }_{x}$ SL sublayers [20]. Table I summarizes the $\mathrm{GaN}_{x} \mathrm{As}_{1-x}$ SL sublayer thicknesses obtained from our ellipsometry investigations in the NIR and IR spectral regions. The thicknesses of the GaAs sublayers are constant $(9 \mathrm{~nm})$ for all samples. The samples were measured by IRSE at two angles of incidence $\left(60^{\circ}, 70^{\circ}\right)$, and for wavenumbers from $250 \mathrm{~cm}^{-1}$ to $700 \mathrm{~cm}^{-1}$ with $2 \mathrm{~cm}^{-1}$ resolution. A commercially available (J.A.Woollam Co.) rotatingcompensator, rotating-polarizer, Fourier-transformbased variable-angle-of-incidence spectroscopic ellipsometer was used [28].

\subsection{Results and Discussion}

Figure 2 presents experimental (symbols) and calculated (solid lines) $\Psi$ spectra from all three samples $\left(\Phi_{a}=\right.$ $\left.70^{\circ}\right)$. Presentation of further angle-of-incidence data is omitted here for clarity. Vertical lines indicate the GaAs $\omega_{\mathrm{TO}}$ and $\omega_{\mathrm{LO}}$ frequencies. The best-fit calculations were performed considering the layered structure of the samples including the SL sequence. The dielectric functions of the constituents were calculated using the dielectric function model and the Drude approximation mentioned above. For the $\mathrm{GaN}_{x} \mathrm{As}_{1-x} \mathrm{SL}$ sublayers we included an additional harmonic oscillator to account for the Ga-N sublattice vibration $\left(\omega_{\mathrm{TO} 2}, \omega_{\mathrm{LO} 2}, \Gamma_{2}\right)$. For the Te-doped $n$-type GaAs substrates we assumed an effective mass parameter of 0.063 free electron mass units, and we obtained an optical mobility parameter of $\mu=3020 \mathrm{~cm}^{2}$ / Vs. The logarithms of the substrate free-carrier concentrations (in units of $\mathrm{cm}^{-3}$ ) obtained from the IRSE data analysis where $17.425 \pm 0.004,17.395 \pm 0.005$ and $17.490 \pm 0.003$ for samples with $x=0.9,1.3$ and $3.3 \%$, respectively. We found that the GaAs phonon frequencies are the same for all sample constituents, even for the $\mathrm{GaN}_{x} \mathrm{As}_{1-x}$ SL sublayers, except for the broadening parameters $\left(\Gamma=1.8,2.5\right.$ and $3.9 \mathrm{~cm}^{-1}$ for $x=0.9 \%, 1.3 \%$ and $3.3 \%$, respectively) of the $\mathrm{GaN}_{x} \mathrm{As}_{1-x}$ sublayers, which increase with increasing $x$. The increased number of dislocation and defects within the $\mathrm{GaN}_{x} \mathrm{As}_{1-x}$ SL sublayers may explain the latter. Because the nitrogen incorporation within the $\mathrm{GaN}_{x} \mathrm{As}_{1-x}$ sublayers is very small, the Ga-N vibration has a small amplitude (i.e., $\left.\left[\omega_{\mathrm{LO} 2}-\omega_{\mathrm{TO} 2}\right] / \omega_{\mathrm{TO} 2} \ll 1\right)$ whereas the Ga-As resonance is almost unchanged. The best-fit calculations shown through Figures 2, 3, 45 and 5 are obtained from the best-fit parameters given in Tables 1 and 2 . The bestfit GaAs model parameters are $\omega_{L O}=291.7 \mathrm{~cm}^{-1}, \omega_{T O}$ $=267.8 \mathrm{~cm}^{-1}$, and $\varepsilon_{\infty}=11.7$. The high-frequency dielec- tric constant for the $\mathrm{GaN}_{x} \mathrm{As}_{1-x}$ SL sublayers were found as $\varepsilon_{\infty}=10.0$.

The Ga-N sublattice vibration resonance, observed within the IR-SE data at $\sim 470 \mathrm{~cm}^{-1}$, and labeled as " $\mathrm{TO}_{2}$ " in Figures 2 and 3, provides sensitivity to the nitrogen concentration within the $\mathrm{GaN}_{x} \mathrm{As}_{1-x}$ sublayers, which is further discussed below. The excitation of the BP (Berreman polariton) causes the dip within all data sets near the GaAs $\omega_{\mathrm{LO}}$ frequency, and provides sensitivity to the buffer-layer and GaAs SL sublayer thicknesses and phonon frequencies. We also observe a sharp resonance structure near $\omega \sim 306 \mathrm{~cm}^{-1}$. This resonance, labeled by $\mathrm{PSP}^{+}$in Figures 2 and 3, is related to the occurrence of the upper-branch PSP TM mode between the GaAs substrate and the combined GaAs buffer layer/ $\mathrm{GaAs} / \mathrm{GaN}_{x} \mathrm{As}_{1-x}$ SL heterostructure film.

Figure 3 shows the complex index of refraction $N+$ $i k=\sqrt{ } \varepsilon$ of the $n$-type GaAs substrate, the GaAs buffer layer (which is identical to the GaAs SL sublayers), and the $\mathrm{GaN}_{0.009} \mathrm{As}_{0.991}$ layer (Sample GaNAs016, see Tables 1 and 2). The position at which the substrate index of refraction $N$ is less than 1 is indicated by a vertical line, and labeled by $\mathrm{PSP}^{+}$. This spectral position matches exactly the frequency at which we observe the resonance feature within our IRSE data on all samples labeled by $\mathrm{PSP}^{+}$in Figure 2. It further matches the condition mentioned above for existence of the unbound TM wave propagating along the substrate/film interface, and its frequency can be exactly located in Figure 1 for the GaAs substrate carrier concentration of $\log \left(n\left[\mathrm{~cm}^{-3}\right]\right)$ $=17.425$. As can be seen in Figure 2, the $\mathrm{PSP}^{+}$resonance is sharp for the sample with $x=0.9 \%$, and less pronounced for the sample with $1.3 \%$. For the sample with $x=3.3 \%$ the $\mathrm{PSP}^{+}$resonance is almost subsumed by the GaAs reststrahlen band, but still present as a weak shoulder on the high-energy side of the GaAs TO$\mathrm{LPP}^{+}$reflectivity band. This damping behavior is not due to the slight increase of the lattice resonance broadening within the $\mathrm{GaAs} / \mathrm{GaN}_{x} \mathrm{As}_{1-x}$ SL heterostructure (See Table 2). In order to successfully model the damping of the $\mathrm{PSP}^{+}$feature we need to consider free-carrier contributions to the optical response of the $\mathrm{GaN}_{x} \mathrm{As}_{1-x}$ SL sublayers. A simple explanation for the damping of the PSP feature is that free carriers within the $\mathrm{GaN}_{x} \mathrm{As}_{1-}$ $x$ SL sublayers screen the incident electromagnetic fields, which otherwise penetrate through the film into the substrate/film interface region. The carrier absorption within the $\mathrm{GaN}_{x} \mathrm{As}_{1-x}$ SL sublayers therefore effectively suppresses the excitation of the $\mathrm{PSP}^{+}$resonance. 
We obtain from our best-fit analysis that the carrier concentration parameter increases with increasing $x$ (Table 2 ), in accordance with the observation of the PSP damping in Figure 2. For the $\mathrm{GaN}_{x} \mathrm{As}_{1-x}$ sublayers we assumed the GaAs effective mass parameter. The optical mobility parameter of $\mu \sim 100 \mathrm{~cm}^{2} /$ Vs obtained for the $\mathrm{GaN}_{x} \mathrm{As}_{1-x}$ SL sublayers is much less than that for the Te-doped substrate. This may indicate holes as majority carriers, which are known to obey smaller mobility. However, from this optical experiment one cannot differentiate between $n$ - or $p$-type conductivity. As discussed in Ref. [18], the free-carrier related quantities derived from the IRSE experiments are the ratios $m^{*} / n$, and $1 /(\mu n)$. Here we assumed the GaAs electron effective mass $m^{*}$, and obtained $n$ given in Table 2, and $\mu \sim 100 \mathrm{~cm}^{2} / \mathrm{Vs}$. Concentration and mobility would change accordingly if the hole effective mass parameter would be chosen for data analysis, but the calculated best-fit spectra as well as all other parameters would remain unchanged.

Figure 4 presents the enlarged section for the Ga-N sublattice resonance frequency $\left(\mathrm{TO}_{2}\right)$ within our IRSE data (Figure 2). The vertical line indicates the $\mathrm{TO}_{2}$ resonance. The frequency shift of $\mathrm{TO}_{2}$ versus $x$ (see Tab. II) is negligible, and within its uncertainty limit of \pm 0.5 $\mathrm{cm}^{-1}$. On the other hand, the TO-LO splitting of the Ga$\mathrm{N}$ resonance, $f=\left(\omega_{T O^{-}} \omega_{L O}\right) / \omega_{T O}$, i.e., the polar strength of the Ga-N phonon branch, increases with increasing $x$. We obtain that $f$ increases linearly versus $x$. We also observe an increase in $\Gamma_{2}$ with $x$ due to the increase in dislocation and disorder within the $\mathrm{GaN}_{x} \mathrm{As}_{1-x}$ SL sublayers. Accordingly, the forbidden second harmonic of the GaAs LO frequency $2 \mathrm{LO}_{1}$ is detected within the IRSE data from the sample with $x=3.3 \%$. This observation is explained by the breakdown of selection rules in the $\mathrm{GaN}_{x} \mathrm{As}_{1-x}$ sublayers due to the increase of straininduced lattice disorder.

Figure 5 shows the dependences of the relative $\mathrm{Ga}-\mathrm{N}$ resonance LO-TO splitting $f$ and the $\mathrm{GaN}_{x} \mathrm{As}_{1-x}$ sublayer carrier concentration $n$ versus $x$. The linear dependence of $f$ versus $x$ obtained here can be used to test the nitrogen concentration in other $\mathrm{GaN}_{x} \mathrm{As}_{1-x}$ epilayers. Note that the sensitivity of the IR-SE data to $n$ is larger for higher concentration $x$ because free-carrier detection limits exist for small concentration values [18], [22].

\section{Conclusion}

In this report we present and discuss room temperature IRSE data taken from GaAs/GaN ${ }_{x} \mathrm{As}_{1-x}$ SL structures grown by MOVPE. We obtain that the $\mathrm{GaN}_{x} \mathrm{As}_{1-x} \mathrm{SL}$ sublayers contain free carriers, which may originate from misfit-dislocation-induced or nitrogen-induced donor or acceptor states within the SL structure. We report the optically determined free-carrier concentrations within the substrate and the $\mathrm{GaN}_{x} \mathrm{As}_{1-x}$ sublayers, and the frequency, broadening, and amplitude of the nitride-impurity lattice mode as a function of the nitrogen concentration $x$ for $0 \leq x \leq 3.3 \%$. We discuss the origin of the resonance structures within the IR-SE data, and assign the excitation of pseudo surface polaritons between the doped GaAs substrate and the GaAs/ GaNAs heterostructure as the cause for the experimental observation. We found that the pseudo surface polariton excitation depends crucially on the screening mechanism within the SL heterostructure, and therefore provides sensitivity to free-carrier properties within the $\mathrm{GaN}_{x} \mathrm{As}_{1-x}$ SL sublayers.

\section{ACKNOWLEDGMENTS}

This work is supported by the Deutsche Forschungsgemeinschaft under grants GO 629/3-1 and $R h$ 28/3-1, and by the National Science Foundation contract DMI-9901510. The Center for Microelectronic and Optical Materials Research at University of Nebraska-Lincoln, and the Laboratory of Thin Films and Nanostructures at Department of Solid State Physics of Masaryk University Brno further supported this work.

\section{REFERENCES}

[1] M Kondow, K Uomi, T Kitatani, S Watahiki, Y Yazawa, J. Cryst. Growth 164, 175-179 (1996).

[2] Su-Huai Wei, Alex Zunger, Phys. Rev. Lett. 76, 664-667 (1996).

[3] Jorg Neugebauer, Chris G. Van de Walle, Phys. Rev. B 51, 10568-10571 (1995).

[4] A Ougazzaden, YL Bellego, EVK Rao, M Juhel, L Leprince, G Patriarche, Appl. Phys. Lett. 70, 2861-2863 (1997).

[5] E. V. K. Rao, A. Ougazzaden, Y. Le Bellego, M. Juhel, Appl. Phys. Lett. 72, 1409 (1998).

[6] WG Bi, CW Tu, Appl. Phys. Lett. 70, 1608-1610 (1997).

[7] S. Francoeur, G. Sivaraman, Y. Qiu, S. Nikishin, H. Temkin, Appl. Phys. Lett. 72, 1857 (1998).

[8] K. Uesugi, I. Suemune, Jpn. J. Appl. Phys. 36, L1572 (1997).

[9] K. Onabe, D. Aoki, J. Wu, H. Yaguchi, Y. Shiraki, Phys. Stat. Sol. A 176, 231 (1999).

[10] I. A. Buyanova, W. M. Chen, G. Pozina, J. P. Bergman, B. Monemar, H. P. Xin, C. W. Tu, Appl. Phys. Lett. 75, 501 (1999).

[11] A. M. Mintairov, P. A. Blagnov, V. G. Melehin, N. N. Faleev, J. L. Merz, Y. Qiu, S. A. Nikishin, H. Temkin, Phys. Rev. B 56, 15836 (1997).

[12] T. Prokofyeva, T. Sauncy, M. Seon, M. Holtz, Y. Qiu, S. Nikishin, H. Temkin, Appl. Phys. Lett. 73, 1409 (1998). 
[13] A. Roeseler, Infrared Spectroscopic Ellipsometry, (Akademie-Verlag, Berlin 1990)

[14] D. W. Berreman, Phys. Rev. 130, 2193 (1963).

[15] J. Humlicek, Phys. Stat. Sol. B 215, 155 (1999).

[16] St. Zollner, J. P. Carrejo, T. E. Tiwald, J. A. Woollam, Phys. Stat. Sol. B 208, R3 (1998).

[17] M. Schubert, A. Kasic, T. E. Tiwald, J. A. Woollam, V. Härle, F. Scholz, "Phonons and free carriers in a strained hexagonal GaN-AlN superlattice measured by Infrared Ellipsometry and Raman spectroscopy", Mater. Res. Soc. Symp. Proc. 595, W11.39 (2000)

[18] M. Schubert, A. Kasic, T.E. Tiwald, J. Off, B. Kuhn, Ferdinand Scholz, MRS Internet J. Nitride Semicond. Res. 4, 11 (1999).

[19] M. Schubert, J. A. Woollam, A. Kasic, B. Rheinländer, J. Off, B. Kuhn, F. Scholz, Phys. Stat. Sol. B 216, 655 (1999).

[20] J. Sik, M. Schubert, G. Leibiger, V. Gottschalch, G. Kirpal, J. Humlícek, "Near-band-gap optical constants spectra and band-gap energies of GaNAs/GaAs superlattice heterostructures measured by spectroscopic ellipsometry", Appl. Phys. Lett., in press (2000)

[21] M. Schubert, T. E. Tiwald, C. M. Herzinger, Phys. Rev. B 61, 8187 (2000).

[22] A. Kasic, M. Schubert, T.E. Tiwald, J.A. Woollam, S. Einfeldt and D. Hommel, "Free-carrier and phonon properties of n- and p-type hexagonal GaN films measured by Infrared Ellipsometry", unpublished.

[23] A. A. Kukharskii, Sol. St. Comm. 13, 1761 (1973).

[24] V. M. Agranovich, D. L. Mills (editors) Surface Polaritons (North-Holland, Amsterdam, 1982), p.10

[25] M. Schubert, T. Hofmann, T. E. Tiwald, J. Sik, unpublished.

[26] S. S. Mitra, "Optical Properties of Nonmetallic Solids for Photon Energies below the fundamental Band Gap", in E. D. Palik, Handbook of Optical Constants of Solids, (Academic Press, San Diego, 1998), Vol. I, pp. 213-270.

[27] M. Schubert, B. Rheinländer, E. Franke, H. Neumann, T. E. Tiwald, J. A. Woollam, J. Hahn, F. Richter, Phys. Rev. B 56, 13306 (1997).

[28] D. W. Thompson, M. J. DeVries, T. E. Tiwald, J. A. Woollam, Thin Sol. Films 313, 341 (1998).
FIGURES

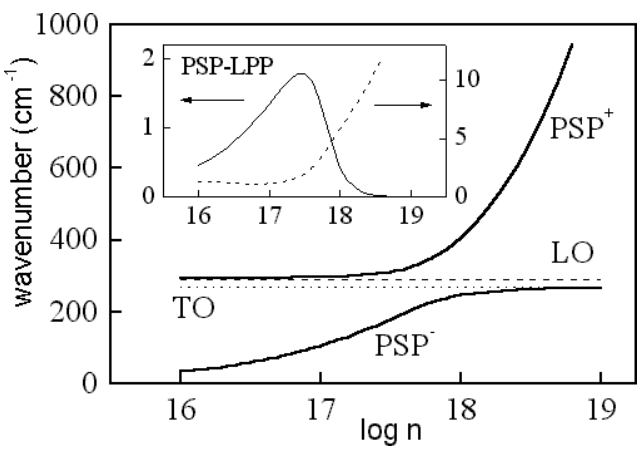

Figure 1. Dispersion of pseudo surface polariton modes at the interface doped / undoped GaAs as a function of the free-carrier concentration $n$ for $k_{x}=\sin \left(70^{\circ}\right)$. The two branches $\left(\mathrm{PSP}^{+}\right.$, $\mathrm{PSP}^{-}$) follow closely those of the longitudinal-optical coupled plasmon-phonon bulk modes $\left(\mathrm{LPP}^{+}, \mathrm{LPP}^{-}\right)$. The horizontal lines indicate the TO and LO frequencies of GaAs. The inset displays the difference between the PSP and LPP modes ( $\mathrm{PSP}^{+}$$\mathrm{LPP}^{+}$: right axis, $\mathrm{PSP}^{-}-\mathrm{LPP}^{-}$: left axis). Parameters used for calculations are $\omega_{L O}=291.7 \mathrm{~cm}^{-1}, \omega_{T O}=267.8 \mathrm{~cm}^{-1}, \varepsilon_{\infty}=11.7$, $m^{*}=0.063 m_{e}$. No broadening is assumed here for simplicity. 


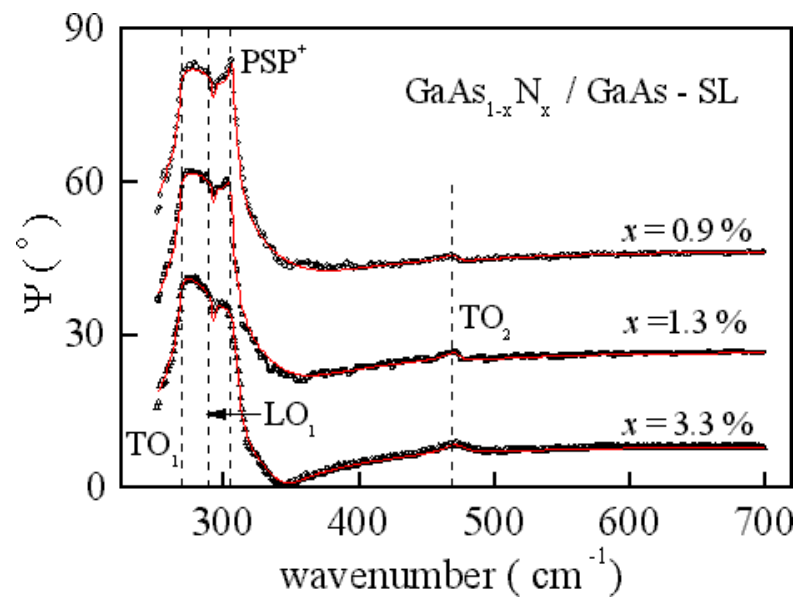

Figure 2. Experimental data (symbols), and best-fit calculation (solid lines) of $\Psi$ from $\mathrm{GaAs} / \mathrm{GaN}_{x} \mathrm{As}_{1-x}$-SL heterostructures (See Table 1). Vertical lines indicate the GaAs $\omega_{\mathrm{TO}}\left(\mathrm{TO}_{1}\right)$ and $\omega_{\mathrm{LO}}\left(\mathrm{LO}_{1}\right)$ frequencies. The Berreman-polariton effect above the GaAs $\mathrm{LO}_{1}$ frequency causes distinct resonance features in all samples. The upper-branch $\mathrm{PSP}^{+}$mode is excited near $306 \mathrm{~cm}^{-1}$ between the $n$-type GaAs substrate and the undoped GaAs buffer layer / SL heterostructure. See also Figure 3. The angle of incidence is $\Phi_{a}=70^{\circ}$. Spectra are to scale, but shifted for convenience by $20^{\circ}$ each. The $\mathrm{PSP}^{+}$resonance becomes washed out for higher nitrogen contents due to screening of the incident electromagnetic field components within the $\mathrm{GaN}_{x} \mathrm{As}_{1-x}$-SL sublayers by free carriers, which concentrate within the $\mathrm{GaN}_{x} \mathrm{As}_{1-x}$-SL sublayers. At $\mathrm{TO}_{2}$, the Ga-N sublattice vibration is detected within the IRSE spectra. See also Figure 4.

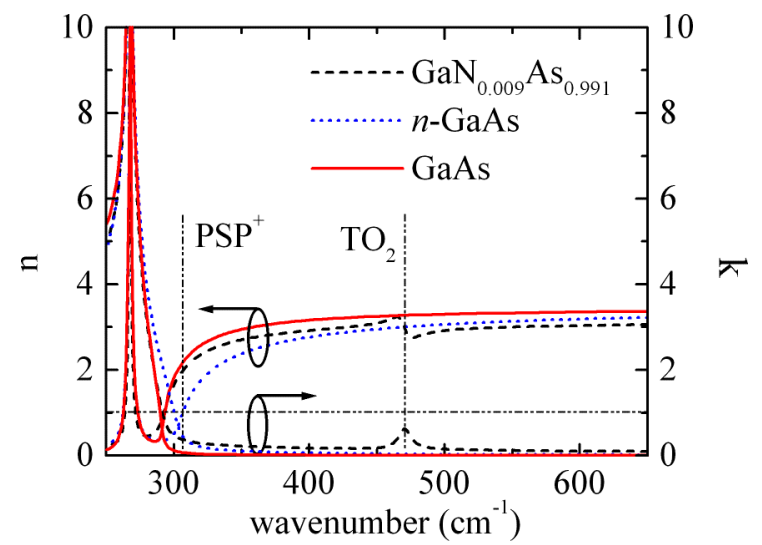

Figure 3. Index of refraction $N$ and extinction coefficient $k$ ( $\varepsilon=$ $[N+i k]^{2}$ ) of the $n$-type GaAs substrate (dotted lines), the undoped GaAs buffer layer and SL sublayers (solid lines), and $\mathrm{GaN}_{0.009} \mathrm{As}_{0.991}$ (dashed line) for example. The position of the $\mathrm{PSP}^{+}$mode is indicated as the frequency at which the substrate refractive index becomes less than 1 . The $\mathrm{GaN}_{0.009} \mathrm{As}_{0.991}$ optical spectra reveal the Ga-N sublattice resonance at $\mathrm{TO}_{2}$ (vertical line).

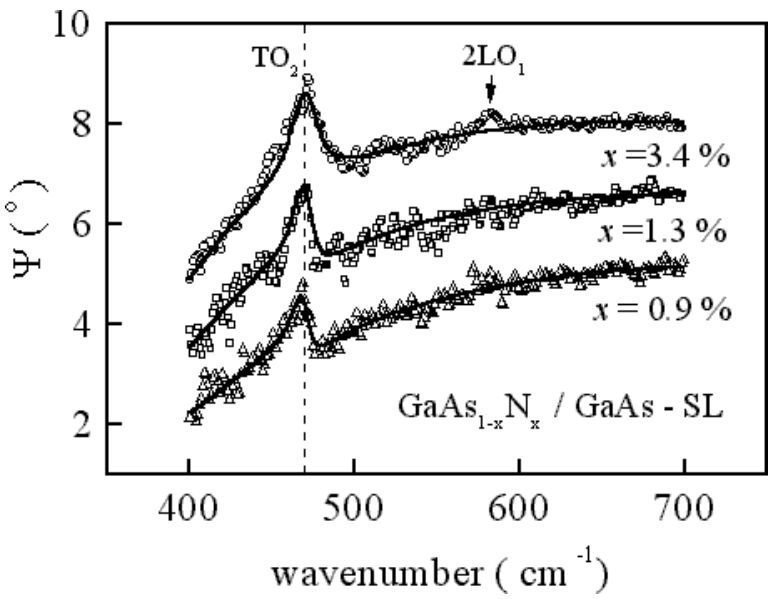

Figure 4. Enlarged section of Figure 2 within the Ga-N sublattice resonance frequency $\left(\mathrm{TO}_{2}\right)$ range. (Spectrum for $x=0.9 \%$ is shifted by $-1^{\circ}$ for convenience.) The feature labeled $2 \mathrm{LO}_{1}$ is assigned as the forbidden but disorder-activated second harmonic of the GaAs LO frequency in sample GaNAs019.

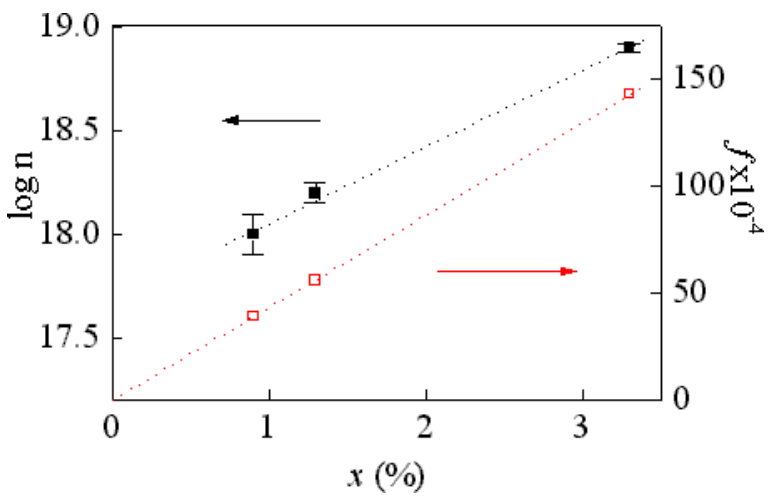

Figure 5. $\mathrm{GaN}_{x} \mathrm{As}_{1-x}$ relative TO-LO splitting $f$ and carrier concentration $n$ versus $x$ obtained from IRSE data analysis (See Table 2; Lines are drawn to guide the eye.). 


\section{TABLES}

Table 1. Sample parameters obtained from XRD $(x)$, NIRSE $\left(\mathrm{d}_{\mathrm{NIR}}\right)$ and IRSE $\left(\mathrm{d}_{\mathrm{IR}}\right)$ investigations. The error bars for all thicknesses amount to $10 \%$, and correspond to $90 \%$ reliability.

\begin{tabular}{|l|l|l|l|}
\hline sample & $x[\%]$ & $d_{\mathrm{IR}}[\mathrm{nm}]$ & $\mathrm{d}_{\mathrm{NIR}}[\mathrm{nm}]$ \\
\hline GaNAs016 & 0.9 & 7.7 & 7.5 \\
\hline GaNAs014 & 1.3 & 8.4 & 7.7 \\
\hline GaNAs019 & 3.3 & 5.0 & 5.1 \\
\hline
\end{tabular}

Table 2. Best-fit parameters for $\mathrm{GaN}_{x} \mathrm{As}_{1-x}(0 \leq x \leq 3.3 \%$; See text for further details).

\begin{tabular}{|l|l|l|l|l|l|}
\hline sample & $x[\%]$ & $n\left[\mathrm{~cm}^{-3}\right] \times 10^{18}$ & $\omega_{\text {Tо2 }}\left[\mathrm{cm}^{-1}\right]$ & $\Gamma\left[\mathrm{cm}^{-1}\right]$ & $f \times 10^{-4}$ \\
\hline GaNAs016 & 0.9 & 1 & 470.0 & 11 & 39 \\
\hline GaNAs014 & 1.3 & 1.6 & 470.7 & 13 & 56 \\
\hline GaNAs019 & 3.3 & 7.9 & 470.9 & 18 & 143 \\
\hline
\end{tabular}

